\title{
Risk factors for developing high-output ileostomy in CRC patients: a retrospective study
}

\author{
Dongxiao Bai ${ }^{\dagger}$, Lei Li ${ }^{\dagger}$, Zhiling Shen, Tianchen Huang, Qingbing Wang, Yanjun Wang, Yong Zhang, \\ Zhipeng Guo, Kan Li and Jian an Xiao ${ }^{*}$ (])
}

\begin{abstract}
Background: Anastomotic leakage is one of the most serious postoperative complications of rectal cancer. Prophylactic ileostomy has been widely used to reduce the risk and severity of complications of anastomotic leakage. However, prophylactic ileostomy itself has some complications, and ileostomy high output syndrome (HOS) is one of them. This study was performed to explore the risk factors of HOS in ileostomy.

Methods: A total of 114 patients with HOS were screened out from 494 eligible ileostomy patients in the last 5 years. The relationship between HOS and the clinicopathological data was analyzed using the Chi-square test and Fisher's exact probability. Multivariate analysis was performed by logistic regression.

Results: The incidence of HOS was $23.07 \%$ in this study. Dehydration was the most common symptom of HOS (37.7\%). There was no clear correlation between HOS occurrence with sex, age, gross typing, histological grade, tumor location, lymph node metastasis, and TNM stage ( $p>0.05)$. The incidence of HOS was 14/18 in inflammatory bowel disease patients, 18/28 in diabetes mellitus patients, and 23/72 in neoadjuvant chemoradiotherapy patients, 13/17 in total colectomy and abdominal infection patients. Multivariate analysis showed that they are risk factors for HOS $(p<0.05)$.
\end{abstract}

Conclusion: HOS occurred occasionally but rarely studied and lacks attention. Inflammatory bowel disease, diabetes mellitus, neoadjuvant radiotherapy chemotherapy, total colectomy and abdominal infection are the risk factors for HOS.

\section{Background}

Among Chinese, colorectal cancer (CRC) is the fourth most common tumor in men and women. It accounts for $10.46 \%$ of all new cancer cases in men and $9.17 \%$ in women. Furthermore, the incidence of colorectal cancer is increasing [1]. Fortunately, more standardized and individualized treatments have reduced mortality and improved CRC patients' quality of life. Radical surgery is still the primary means of treatment, but some patients with low rectal cancer have anastomotic fistula after

\footnotetext{
*Correspondence: xiaojawsk@126.com
}

${ }^{\dagger}$ Dongxiao Bai and Lei Li contributed equally to this work

Department of Surgery, Anyang Tumor Hospital, No. 1 Huanbin North

Road, Anyang 455000, Henan, China surgery. Prophylactic ileostomy has been widely used to reduce the risk and severity of complications of anastomotic leakage $[2,3]$.

In general, ileostomy indications include colon injury, colorectal cancer, familial polyposis, ulcerative colitis, crohn's disease, etc. For some advanced colorectal cancer patients with obstruction, it is necessary first to remove the intestinal obstruction with an ostomy, or an ileostomy is also feasible to carry out adjuvant therapy. For some middle and low rectal cancer and left colon cancer, edema, or others with high-risk factors of anastomotic fistula, it may be necessary to adopt preventive ileostomy to reduce the risk and complications of anastomotic leakage when resecting the lesion and one-stage anastomosis [4]. We generally agreed that preventive (prophylactic) 
ileostomy should be undergone on patients with the following situations. Patients underwent preoperative radiotherapy, especially those intestinal wall texture is brittle, with obvious congestion and edema; Low and ultra-low anastomosis, especially for those less than $3 \mathrm{~cm}$. Poor proximal intestinal blood supply; Unsatisfactory anastomosis, large intestinal tension after anastomosis or positive intraoperative inflatable test; Age greater than 73 years old, poor cardiopulmonary function, malnutrition, anemia, and chronic persistent obstruction patient or long duration; Diabetes patients with poor blood glucose control, the long-term use of hormone.

However, prophylactic ileostomy itself has some complications, and ileostomy high output syndrome is one of them. The risk factors and predictors of high-output ileostomy have been reported in studies with a small sample size $[5,6]$. We conducted a single-institution retrospective review of CRC patients who underwent ileostomy (both prophylactic and therapeutic) in our hospital, and the clinical and pathological data were analyzed to explore the risk factors of high-output ileostomy.

\section{Methods}

The patients with colorectal cancer who underwent ileostomy for 5 consecutive years from March 2013 to March 2018 were collected in Anyang Tumor Hospital of Henan province, China. Patients enrolled were between 26 and 87 years old, with complete medical records, and were followed up either with ileostomy reversal or more than 1 year after ileostomy. HOS was defined as patients whose daily stoma output is more than $2000 \mathrm{ml}$ and lasted more than 2 days, as a previous study reported [7]. The others were excluded from the group whose stoma discharge was not detailed and did not meet the followup requirements. One hundred fourteen patients (63 males and 51 females) with HOS were screened out from 494 eligible ileostomy patients in the last 5 years.

Among the 494 patients with enterostomy, 343 patients (68.6\%) underwent preventive ileostomy during anterior resection (AR) or low anterior resection (LAR), 26 patients underwent ileostomy for bowel obstruction, 17 patients underwent a total colon resection. Emergency operations were performed on five patients. There were 1319 rectal operations cases during 2013-2018, and 343 patients underwent preventive ileostomy (accounting for $26.0 \%)$.

Early HOS (EHOS) was defined as HOS developed within 3 weeks after the operation, and late HOS (LHOS) was those over 3 weeks.

We collected clinical data using patient medical records, outpatient follow-ups, pathological stages, and complications like stoma discharge, electrolyte imbalance, dehydration, and disease treatments. Discharged patients were followed up to 1 year after ileostomy or ileostomy reversal.

SPSS17.0 statistical software was used for the statistical analysis; single-factor analysis was performed using the Chi-square test and Fisher's exact probability method. The multifactor analysis was performed with logistic regression. $\mathrm{p}<0.05$ had statistical significance.

\section{Results}

1. Common symptoms of HOS. Dehydration was the most common symptom of HOS (37.7\%). Those patients need rehydrate with intravenous fluid or additional anti-secretory (somatostatin)/diarrheal (loperamide) medication treatment are $29.8 \%$ and $7.9 \%$ respectively (Fig. 1). Followed symptoms are electrolyte disturbance (28.1\%), local dermatitis (21.9\%), renal dysfunction (5.3\%), and malnutrition (7.0\%) in long-term HOS (Table 1).

2. Correlation between HOS and clinicopathological features of CRC. The results showed no clear correlation between the occurrence of HOS with gender, age, gross typing, histological grading, tumor location, lymph node metastasis, and TNM staging in patients with colorectal cancer (Table 2).

3. Correlation between HOS and preoperative treatments and concomitant diseases. These data showed that patients with inflammatory bowel disease, diabetes mellitus, and neoadjuvant chemoradiotherapy are associated with HOS occurrence (Table 3).

4. HOS and surgical-related indicators. These figures showed that total colectomy and postoperative abdominal infection are correlated with the occurrence of HOS but not with operation time, bleeding volume, application of diuretics, and laparoscopy (Table 4).

5. EHOS/LHOS and related pathological factors. The study demonstrated there is no significant difference between the occurrence of HOS and related pathological factors (inflammatory bowel disease, diabetes, mellitus, hypoproteinemia, neoadjuvant chemotherapy, anemia, etc.) in ileostomy patients (Table 5).

6. Multivariate analysis of related factors with HOS. The indicators related to the occurrence of HOS in univariate analysis were further analyzed by multivariate analysis. This study showed that preoperative situations including inflammatory bowel disease, diabetes mellitus and neoadjuvant chemoradiotherapy were risk factors for HOS $(\mathrm{p}<0.05)$ (Table 6). 
Dindo Clavien Classification of Dehydration
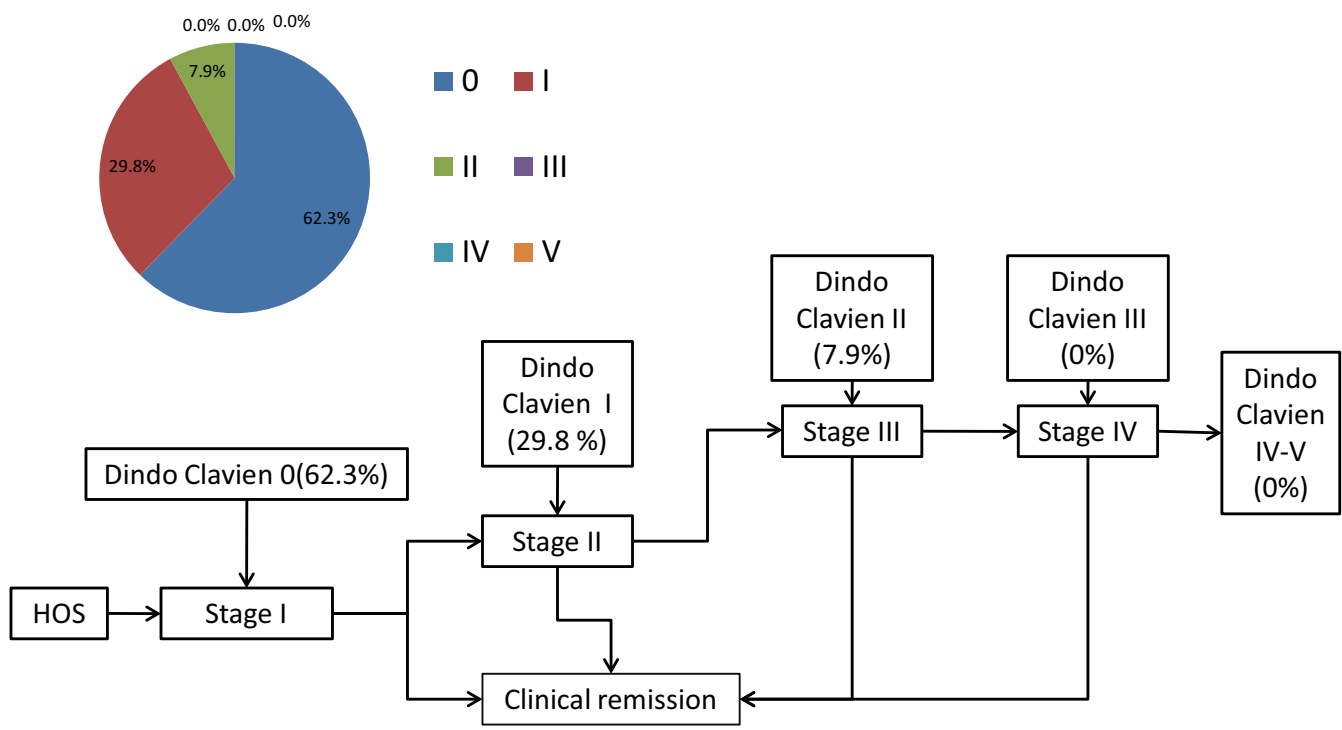

Fig. 1 Management protocol and outcome of HOS patients. STAGE I: exclude potential causes. STAGE II: Initial management-reduce fluid and electrolyte losses. STAGE III: Ongoing HOS - optimise treatment with anti-secretory (somatostatin)/diarrheal (loperamide) medication. STAGE IV: Evaluate efficacy of additional treatment if HOS continues (Adapted from Baker et al. [7], Franklin Adaba et al. [17])

Table 1 Composition ratio of common symptoms of HOS

\begin{tabular}{lll}
\hline HOS & $(\mathbf{n = 1 1 4 )}$ & Proportion (\%) \\
\hline Dehydration & 43 & 37.7 \\
Electrolyte disturbance & 32 & 28.1 \\
Hyponatremia & 21 & 18.4 \\
Hypochloritemia & 18 & 15.8 \\
Hypokalemia & 14 & 12.3 \\
Hypomagnesemia & 4 & 3.5 \\
Renal dysfunction & 6 & 5.3 \\
Malnutrition & 8 & 7.0 \\
Local dermatitis & 25 & 21.9 \\
\hline
\end{tabular}

\section{Discussion}

HOS or high-output syndrome (basically the same) is rarely studied and lacks attention. There is no consensus on the definition of HOS [5, 7-10]. Dehydration, electrolyte disturbance (hyponatremia, hypochloremia, hypomagnesemia), renal failure, and malnutrition (latestage) can occur in high-output ileostomy $[5,7,8]$. HOS increases the risk of readmission of CRC patients. Some scholars defined HOS as stoma output of more than $1500 \mathrm{ml}$ per day for more than 2 consecutive days $[8,9]$, while the others advocate that HOS should be defined as the output of more than $2000 \mathrm{ml}$ per day for more than 2 or 3 consecutive days $[5,7]$. As the complications are more likely to occur when the daily stoma output exceeds
$2000 \mathrm{ml}$, we adopt the latter standard in our study. HOS can be classified as early $(<3$ weeks after initial ostomy surgery) or late HOS (3 weeks after surgery), and previous studies widely accept this.

In our cohort, the incidence of HOS (114/494) was 23.07\%, higher than earlier reports (17\%) [7]. Dehydration was the most common symptom of HOS (37.7\%), followed by electrolyte disturbance (28.1\%), local dermatitis (21.9\%), renal dysfunction (5.3\%), and malnutrition $(7.0 \%)$ in long-term HOS. It was reported that the incidence of $\mathrm{HOS}$ is $26 \%$ in 262 patients with ileostomy during hospitalization, $30 \%$ of patients were re-admitted within 30 days after discharge, and $37 \%$ of patients with re-hospitalization were due to dehydration [11]. The readmission risk of ileostomy patients with inflammatory bowel disease was double that of other risk factors (OR 2.04) [12].

Our study found no clear correlation between the occurrence of HOS and gender, age, gross typing, histological grading, location of tumors, lymph node metastasis, and TNM staging in patients with colorectal cancer. Preoperative complications of inflammatory bowel disease, diabetes mellitus, and neoadjuvant chemoradiotherapy are risk factors for HOS. Inflammation caused by an abnormal reaction of the intestinal mucosal immune system act as an important role in the pathogenesis of inflammatory bowel disease and is also the leading cause of HOS. Diabetes mellitus occurred in patients with colorectal cancer because of the disorder of glucose 
Table 2 Relationship between HOS and clinical-pathological features

\begin{tabular}{|c|c|c|c|}
\hline Clinicopathological & Cases & $\begin{array}{l}\text { Indicators HOS } \\
\mathrm{n}(\%)\end{array}$ & $P$ value \\
\hline Gender & & & 0.741 \\
\hline Male & 271 & $61(22.5)$ & \\
\hline Female & 223 & $53(23.8)$ & \\
\hline Age & & & 0.537 \\
\hline$>60$ years old & 193 & $44(22.8)$ & \\
\hline$\leq 60$ years old & 291 & $70(24.1)$ & \\
\hline Gross type & & & 0.726 \\
\hline Massive type & 219 & $53(24.2)$ & \\
\hline Ulcerative type & 162 & $38(23.5)$ & \\
\hline Infiltrating type & 113 & $23(20.4)$ & \\
\hline Organizational grading & & & 0.197 \\
\hline Highly differentiated & 178 & $36(20.2)$ & \\
\hline Medium differentiation & 191 & $42(22.0)$ & \\
\hline Poorly differentiated & 125 & $36(28.8)$ & \\
\hline Tumor site & & & 0.487 \\
\hline Rectum & 351 & $79(22.5)$ & \\
\hline Colon & 143 & $35(24.5)$ & \\
\hline Lymph node metastasis & & & 0.487 \\
\hline Yes & 307 & $74(24.1)$ & \\
\hline No & 187 & $40(21.4)$ & \\
\hline TNM staging & & & 0.934 \\
\hline Phase I & 55 & $13(23.6)$ & \\
\hline Phase II & 85 & $20(23.5)$ & \\
\hline Phase III & 229 & $50(21.8)$ & \\
\hline Phase IV & 125 & $31(24.8)$ & \\
\hline
\end{tabular}

metabolism and utilization. Examples such as inappropriate control, blood sugar, or intestinal movements may result in a large amount of liquid discharged from ileostomy [5]. We found that neoadjuvant concurrent radiotherapy and chemotherapy (CRT) is a risk factor for HOS
(HOS). Radiation enteritis caused by neoadjuvant radiotherapy might be responsible for HOS in preoperative CRC patients [13]. As neoadjuvant chemoradiotherapy is mostly carried out simultaneously before an operation in our hospital, there is no stratified study of neoadjuvant chemotherapy, radiotherapy alone, and neoadjuvant concurrent radiotherapy and chemotherapy. It has been reported that preoperative radiotherapy alone, the distance between the tumor and anal margin, could affect the intestinal function of patients after operation, while chemotherapy alone has no significant effect on intestinal function after operation [14].

Among the related factors of operation, total colectomy and abdominal infection are the risk factors of HOS, but the operation time, bleeding volume, diuretic application, and laparoscopic operation is not. High stoma displacement in inflammatory bowel disease for most IBD patients was complicated with long-term diarrhea before surgery, and we believe that high stoma displacement is mainly related to the primary disease. Long-term oral administration of drugs (non-steroidal anti-inflammatory drugs) and hormone use may also be factors. Diabetes mellitus and total colectomy are high-risk factors for HOS, consistent with a previous study by Takeda and others.[5]. As reported, bile acid deficiency is one of the mechanisms of total colectomy caused by HOS. Total proctocolectomy prevents the reabsorption of bile acids absorbed by the ileocecum. As a result decrease in bile acid pools inhibits lipid absorption. Consequently, unabsorbed long-chain fatty acids are hydroxylated or desaturated by anaerobic intestinal bacteria, triggering the secretion of fluid and electrolytes, which may lead to the development of HOS [5]. Bile acid deficiency may also cause changes in the intestinal flora that increase intestinal drainage [15]. The loss of water absorption by the colon in patients undergoing total colectomy can also lead to high ileostomy output. Our study did not find that

Table 3 Univariate analysis of preoperative situations in HOS and non-HOS groups

\begin{tabular}{|c|c|c|c|}
\hline \multirow[t]{2}{*}{ Complications } & \multicolumn{2}{|l|}{ lleostomy n (\%) } & \multirow[t]{2}{*}{$P$ value } \\
\hline & $\operatorname{HOS}(n=114)$ & Non-HOS $(n=380)$ & \\
\hline Inflammatory bowel disease & $14(12.3)$ & $4(1.1)$ & $<0.01^{*}$ \\
\hline Diabetes & $18(15.8)$ & $10(2.6)$ & $<0.01^{*}$ \\
\hline Mental disorder (tension) & $5(4.4)$ & $15(3.9)$ & 0.84 \\
\hline Intestinal obstruction & $6(5.3)$ & $21(5.5)$ & 0.91 \\
\hline Hypoproteinemia & $11(9.6)$ & $42(11.1)$ & 0.96 \\
\hline Anemia & $6(5.3)$ & $22(5.8)$ & 0.83 \\
\hline Neoadjuvant chemoradiotherapy & $23(20.2)$ & 49 (12.9) & $0.04^{*}$ \\
\hline Steroid hormones & $4(3.5)$ & $6(1.6)$ & 0.18 \\
\hline
\end{tabular}

* The difference was statistically significant 
Table 4 Univariate analysis of surgical related indicators in $\mathrm{HOS}$ and non-HOS groups

\begin{tabular}{|c|c|c|c|}
\hline \multirow[t]{2}{*}{ Clinicopathological indicators } & \multicolumn{2}{|l|}{ lleostomy n (\%) } & \multirow[t]{2}{*}{$P$ value } \\
\hline & $\operatorname{HOS}(n=114)$ & Non-HOS $(n=380)$ & \\
\hline Radical operation & & & $<0.01^{*}$ \\
\hline Radical resection of rectal cancer & $67(58.8)$ & $250(65.8)$ & \\
\hline Radical resection of colon cancer & $34(29.8)$ & $126(33.2)$ & \\
\hline Total colectomy & $13(11.4)$ & $4(1.1)$ & \\
\hline Laparoscopic surgery & & & 0.467 \\
\hline Yes & $81(71.1)$ & $283(74.5)$ & \\
\hline No & $33(28.9)$ & $97(25.5)$ & \\
\hline Operative time (h) & & & 0.352 \\
\hline$\geq 3$ & $61(53.5)$ & $222(58.4)$ & \\
\hline$<3$ & $53(46.5)$ & $158(41.6)$ & \\
\hline Surgical bleeding volume (ml) & & & 0.514 \\
\hline$\geq 300$ & $38(33.3)$ & $115(30.3)$ & \\
\hline$<300$ & $76(66.7)$ & $265(69.7)$ & \\
\hline Postoperative abdominal infection & $8(7.0)$ & $5(1.3)$ & $0.000^{*}$ \\
\hline Postoperative application of Diuretics & $3(2.6)$ & $4(1.1)$ & 0.204 \\
\hline
\end{tabular}

*The difference was statistically significant

Table 5 The relationship between EHOS, LHOS and related pathological factors

\begin{tabular}{llll}
\hline Clinicopathological factors & \multicolumn{2}{l}{ HOS $(\mathbf{n}=\mathbf{1 1 4}) \mathbf{n}(\%)$} & $P$ value \\
\cline { 2 - 3 } & \multicolumn{1}{c}{$\operatorname{EHOS~(n=69)}$} & LHOS $(\mathbf{n}=\mathbf{4 5})$ & \\
\hline Inflammatory bowel disease & $10(14.5)$ & $4(8.9)$ & 0.279 \\
Diabetes & $11(15.9)$ & $7(15.6)$ & 0.956 \\
Mental disorder (tension) & $3(4.3)$ & $2(4.4)$ & 1.000 \\
Hypoproteinemia & $8(11.6)$ & $3(6.7)$ & 0.520 \\
Anemia & $4(5.8)$ & $2(4.4)$ & 1.000 \\
Neoadjuvant chemoradio- & $11(15.9)$ & $12(26.7)$ & 0.163 \\
$\quad$ therapy & $3(4.3)$ & $1(2.2)$ & 0.483 \\
Steroid hormones & &
\end{tabular}

the use of steroids and diuretics after ileostomy increases the high output of ileostomy, but large doses of diuretics may increase the risk of dehydration.

The aims of management of patients with HOS are to: Provide nutrients, electrolyte, and water necessary to maintain health and growth, reduce the severity of intestinal failure, prevent and treat complications due to intestinal failure, achieve a good quality of life [16, 17]. Generally, half of the EHOS patients required no particular drug intervention until recuperation, while the other half required drug intervention [7]. For the treatment and management of HOS, patients should be managed and guided throughout the hospital and after discharge [18]. In addition to basic treatments (such as restricting fluid intake, rehydration, and correcting electrolyte disturbance), reducing the secretion of somatostatin and oral intake of loperamide effectively reduces discharge. Pieter-Jan Cuyle reported that 17\% of patients with ileostomy had high excretion [19]. This complication would affect the implementation or completion of adjuvant therapy. Somatostatin analogues (ranitides, etc.) could reduce the excretion of ileostomy effectively. The routine dosage of loperamide is $20 \mathrm{mg}$ twice daily by mouth, but the dosage can be increased if it is not effective. Alicia Mackowski reported that increasing the dosage of loperamide to $30 \mathrm{mg}$ per day

Table 6 Logistic regression multivariate analysis

\begin{tabular}{lllllcc}
\hline Clinicopathological factors & B & S.E & Wals & Sig & Exp (B) & 95\%Cl \\
\hline Inflammatory bowel disease & 3.517 & .636 & 30.555 & .000 & 33.686 & $9.680-117.229$ \\
Diabetes & 3.061 & .508 & 36.244 & .000 & 21.351 & $7.882-57.841$ \\
Neoadjuvant hemoradiotherapy & 2.149 & .425 & 25.533 & .000 & 8.580 & $3.727-19.749$ \\
Total colectomy & 1.191 & .354 & 11.344 & .000 & 3.291 & $1.646-6.584$ \\
Steroid hormones & 3.470 & .701 & 24.472 & .000 & 32.146 & $8.133-127.060$ \\
\hline
\end{tabular}


in individual patients can reduce the amount of stoma excretion, without observed abnormality in renal function [20]. Unfortunately, we cannot record the specific time of HOS patients' return and only make a classification based on 3 weeks.

This paper is a retrospective study with some limitations. A prospective study should pay attention to the patients with high-risk factors HOS, strengthen the supervision and treatment after the operation and discharge for reducing the complications of stoma, and facilitate the smooth progress of comprehensive treatment and the readmission rate caused by HOS [21-24]. Furthermore, HOS generally increases the length of hospitalization, the cost of hospitalization, and the readmission rate, leading to clinical pathway variation, while details are not yet available. ICU transfer rate and followup survival were not included in the original data of this study.

We followed up with patients who had ileostomy reversal or 1 year after ileostomy by inpatient medical records, outpatient review, and telephone follow-up. These data mostly came from patients' observation records in the hospital (including outpatient review) belonging to onsite data collection. Using remote video conferences to evaluate ileostomy output and taking early intervention measures to improve prognosis is a method worth exploring [25]. Multicenter, large sample randomized controlled studies of HOS should be conducted in the future.

\section{Conclusion}

These results indicated that inflammatory bowel disease, diabetes mellitus, neoadjuvant chemoradiotherapy, total colectomy and abdominal infection are the risk factors for HOS. Such patients should be alert to the occurrence of HOS in perioperative period of CRC.

\section{Abbreviations \\ CRC: Colorectal cancer; HOS: High-output stoma; EHOS: Early high-output stoma; LHOS: Late high-output stoma.}

\section{Acknowledgements}

Not applicable.

\section{Authors' contributions}

JAX, DXB and LL designed the study. ZLS, TCH and YZ collected clinical data. QBW, LL and YJW performed the statistical analysis. DXB and LL wrote the manuscript, JAX reviewed and revised. All authors read and approved the final manuscript.

\section{Funding}

No resources or funding was obtained for this study.

\section{Availability of data and materials}

The datasets used during the current study are available from the corresponding author on reasonable request.

\section{Declarations}

Ethics approval and consent to participate

The study protocol was approved by the ethical committee for scientific research and drug clinical trial of AnYang Tumor Hospital (NO. AZLL022019048190812). The requirement for informed consent was waived due to the retrospective study design.

\section{Consent for publication}

Not applicable.

\section{Competing interests}

The authors declare that they have no competing interests.

Received: 8 April 2020 Accepted: 6 June 2021

Published online: 26 June 2021

\section{References}

1. Chen W, Zheng R, Baade PD, Zhang S, Zeng H, Bray F, et al. Cancer statistics in China, 2015. CA Cancer J Clin. 2016;66(2):115-32.

2. Chudner A, Gachabayov M, Dyatlov A, et al. The influence of diverting loop ileostomy vs. colostomy on postoperative morbidity in restorative anterior resection for rectal cancer: a systematic review and meta-analysis. Langenbecks Arch Surg. 2019;404(2):129-39.

3. Gavriilidis P, Azoulay D, Taflampas P, et al. Loop transverse colostomy versus loop ileostomy for defunctioning of colorectal anastomosis: a systematic review, updated conventional meta-analysis, and cumulative meta-analysis. Surg Today. 2019;49(2):108-17.

4. Glynne-Jones R, Wyrwicz L, Tiret E, et al. Rectal cancer: ESMO Clinical Practice Guidelines for diagnosis, treatment and follow-up. Ann Oncol. 2017:28:iv22-40.

5. Takeda M, Takahashi H, Haraguchi N, et al. Factors predictive of highoutput ileostomy: a retrospective single-center comparative study. Surg Today. 2019;49(6):482-7.

6. Fujino S, Miyoshi N, Ohue M, et al. Prediction model and treatment of high-output ileostomy in colorectal cancer surgery. Mol Clin Oncol. 2017:7(3):468-72

7. Baker ML, Williams RN, Nightingale JM, et al. Causes and management of a HOS. Colorectal Dis. 2011;13(2):191-7.

8. Arenas Villafranca JJ, López-Rodríguez C, Abilés J, et al. Protocol for the detection and nutritional management of HOSs. Nutr J. 2015;9(14):45.

9. Hayden DM, Pinzon MC, Francescatti AB, et al. Hospital readmission for fluid and electrolyte abnormalities following ileostomy construction: preventable or unpredictable? J Gastrointest Surg. 2013;17:298-303.

10. Kwiatt M, Kawata M. Avoidance and management of stomal complications. Clin Colon Rect Surg. 2013;26:112-21.

11. Justiniano CF, Temple LK, Swanger AA, et al. Readmissions with dehydration after ileostomy creation: rethinking risk factors. Dis Colon Rectum. 2018;61(11):1297-305.

12. Christian KE, Jambaulikar GD, Hagan MN, et al. Predictors of early readmission in hospitalized patients with inflammatory bowel disease. Inflamm Bowel Dis. 2017:23(11):1891-7.

13. Waddell $B E$, Rodriguez-Bigas MA, Lee RJ, et al. Prevention of chronic radiation enteritis. J Am Coll Surg. 1999;189:611-24.

14. Quezada-Diaz F, Jimenez-Rodriguez RM, Pappou EP, et al. Effect of neoadjuvant systemic chemotherapy with or without chemoradiation on bowel function in rectal cancer patients treated with total mesorectal excision. J Gastrointest Surg. 2019;23(4):800-7.

15. Nissinen MJ, Gylling H, Javinen HJ, Miettinen TA. Ileal pouch-anal anastomosis, conventional ileostomy and ileorectal anastomosis modify cholesterol etabolism. Dig Dis Sci. 2004;49:1444-53.

16. Nightingale J, Woodward JM, Small Bowel and Nutrition Committee of the British Society of Gastroenterology. Guidelines for management of patients with a short bowel. Gut. 2006;55(Suppl 4):iv1.

17. Adaba F, Carolynne J, et al. Management of intestinal failure: the highoutput enterostomy and enterocutaneous fistula. Clin Colon Rectal Surg. 2017;30(3):215-22. 
18. Goodey A, Colman S. Safe management of ileostomates with HOSs. Br J Nurs. 2016;25(22):S4-9.

19. Cuyle P-J, Engelen A, Moons V, et al. Lanreotide in the prevention and management of high-output ileostomy after colorectal cancer surgery. J Drug Assess. 2018;7(1):28-33.

20. Mackowski A, Chen H-K, Levitt M. Successful management of chronic high-output ileostomy with high dose loperamide. BMJ Case Rep. 2015;2015:bcr2015209411.

21. Gonella F, Valenti A, Massucco P, et al. A novel patient-centered protocol to reduce hospital readmissions for dehydration after ileostomy. Updates Surg. 2019;71(3):515-21.

22. Knowles SR, Cook SI, Tribbick D, et al. Relationship between health status, illness perceptions, coping strategies and psychological morbidity: a preliminary study with IBD stoma patients. J Crohns Colitis. 2013;7(10):471-8.
23. Chen SY, Stem M, Cerullo M, et al. Predicting the risk of readmission from dehydration after ileostomy formation: the dehydration readmission after ileostomy prediction score. Dis Colon Rectum. 2018;61(12):1410-7.

24. Hayden DM, Pinzon MC, Francescatti AB, et al. Hospital readmission for fluid and electrolyte abnormalities following ileostomy construction: preventable or unpredictable. J Gastrointest Surg. 2013;17(2):298-303.

25. Bednarski BK, Slack RS, Katz M, et al. Assessment of ileostomy output using telemedicine: a feasibility trial. Dis Colon Rectum. 2018;61(1):77-83.

\section{Publisher's Note}

Springer Nature remains neutral with regard to jurisdictional claims in published maps and institutional affiliations.
Ready to submit your research? Choose BMC and benefit from:

- fast, convenient online submission

- thorough peer review by experienced researchers in your field

- rapid publication on acceptance

- support for research data, including large and complex data types

- gold Open Access which fosters wider collaboration and increased citations

- maximum visibility for your research: over 100M website views per year

At BMC, research is always in progress.

Learn more biomedcentral.com/submissions 